Vanessa Lemos • Thaddeus K. Graczyk •

Margarida Alves · Maria Luísa Lobo •

Maria C. Sousa - Francisco Antunes - Olga Matos

\title{
Identification and determination of the viability of Giardia lamblia cysts and Cryptosporidium parvum and Cryptosporidium hominis oocysts in human fecal and water supply samples by fluorescent in situ hybridization (FISH) and monoclonal antibodies
}

Received: 18 July 2005 / Accepted: 9 September 2005 / Published online: 9 September 2005

C) Springer-Verlag 2005

\begin{abstract}
In the present study, fluorescent in situ hybridization (FISH) and monoclonal antibodies (MAbs) were evaluated for species-specific detection and viability determination of Giardia lamblia, Cryptosporidium parvum, and Cryptosporidium hominis in human fecal and water supply samples. A total of 50 fecal human samples positive for G. lamblia cysts, 38 positive for C. parvum, and 23 positive for $C$. hominis were studied. Also, 18 water supply samples positive for Giardia spp. and Cryptosporidium spp. by the United States Environmental Protection Agency (USEPA) Method 1623 were studied by FISH and fluorescein isothiocyanate (FITC)-conjugated MAbs. Eighteen percent of the fecal samples parasitologically positive for G. lamblia presented viable and nonviable cysts, and $5 \%$ of those positive for Cryptosporidium spp. presented
\end{abstract}

V. Lemos · M. Alves · M. L. Lobo · F. Antunes · O. Matos ( () Unidade de Protozoários Oportunistas/VIH

e Outras Protozooses,

Unidade de Parasitologia e Microbiologia Médicas (UPMM), Instituto de Higiene e Medicina Tropical,

Rua da Junqueira, 96,

1349-008 Lisbon, Portugal

e-mail: omatos@ihmt.unl.pt

Tel.: +351-21-3652659

Fax: +351-21-3632105

T. K. Graczyk

Department of Environmental Health Sciences

and Department of Molecular Microbiology and Immunology, Bloomberg School of Public Health, Johns Hopkins University,

Baltimore, MD, USA

M. C. Sousa

Laboratório de Microbiologia e Parasitologia,

Centro de Estudos Farmacêuticos, Faculdade de Farmácia,

Universidade de Coimbra,

Coimbra, Portugal

F. Antunes

Departamento de Doenças Infecciosas,

Faculdade de Medicina (Hospital de Santa Maria),

Universidade de Lisboa,

Lisbon, Portugal viable and nonviable oocysts. Of the 18 water supply samples analyzed, $6(33 \%)$ presented Giardia spp. viable and nonviable cysts and $2(11 \%)$ presented viable and nonviable Cryptosporidium spp. oocysts. G. lamblia identification was confirmed by polymerase chain reaction (PCR) and sequencing of the $\beta$-giardin gene in the fecal and water samples found positive by FISH and FITC-conjugated MAbs. C. parvum and Cryptosporidium muris were identified, by PCR and sequencing of the small subunit of ribosomal RNA gene, in seven and one water samples, respectively. Our results confirm that this technique enables simultaneous visualization, species-specific identification, and viability determination of the organisms present in human fecal and water supply samples.

Keywords Giardia lamblia Cryptosporidium parvum . Cryptosporidium hominis · Detection · Viability · Water supply samples · FISH $\cdot$ MAbs

\section{Introduction}

Cryptosporidium spp. and Giardia spp. are pathogenic protozoan parasites with a worldwide distribution that cause diarrheal illness in humans and animals (Matos et al. 1998; Slifko et al. 2000). Waterborne transmission probably plays a major role in the spread of both infections to large amounts of population. Several outbreaks of cryptosporidiosis and giardiosis, with different magnitudes attributed to water supplies contaminated with (oo)cysts, have been reported (Mac Kenzie et al. 1994; Slifko et al. 2000). Potential sources of (oo)cysts in water supplies include wastewater of human origin and feces from many mammalian species, including cattle and beaver (Solo-Gabriele et al. 1998; Ono et al. 2001). Both cysts and oocysts are capable of surviving environmental stresses, and filtration, flocculation, sedimentation, and disinfection, commonly used for water purification, are not completely effective in removing or inactivating them (Tzipori and Ward 2002). 
The immunofluorescent assay currently used does not distinguish between viable and nonviable (oo)cysts. Direct microscopic examination of the internal structure of these infective stages and methods based on the uptake of vital dyes are labor intensive and are not applied for routine analysis.

Since only viable (oo)cysts are of significant interest for public health, it is of great interest for the water industry to have a reliable test for characterization of the viability of waterborne (oo)cysts that detect infections. Recently, the fluorescent in situ hybridization (FISH) technique utilizing fluorescent-labeled complimentary DNA oligonucleotide probes targeted to specific sequences of cell ribosomal RNA (rRNA) has been described (Amann et al. 1995). rRNA provides a unique target for nucleic acid probes, as rRNA target molecules are generally present in high numbers in viable cells and degree of specificity (genus, species, etc.) may be designed (Vesey et al. 1998; Moter and Gobel 2000; Smith et al. 2004). Graczyk et al. 2003, tested the suitability and optimized a protocol for dual labeling, FISH and fluorescein isothiocyanate (FITC)-conjugated monoclonal antibody $(\mathrm{MAb})$ for the specific detection and viability determination of Giardia lamblia, Cryptosporidium parvum, and Cryptosporidium hominis carried by synanthropic flies. Incorporation of FITC-conjugated MAb into the FISH protocol allows observation of the external morphology of pathogens and assessment of any structural damage to their walls that can lead to nonviability.

The aim of this study was to determine the applicability of FISH with MAbs to the detection and viability determination of three protozoa species, G. lamblia, C. parvum, and $C$. hominis, isolated from human fecal and water supply samples. Hybridization protocols were optimized for getting a better concentration of oligonucleotide probe applied and the volume of samples used.

\section{Materials and methods}

Identification and processing of $G$. lamblia cysts and $C$. parvum and $C$. hominis oocysts Fecal samples from patients with diarrhea, obtained between 1997 and August 2004, were analyzed retrospectively: 50 with $G$. lamblia cysts, detected on fecal smears after concentration, followed by Lugol staining and 61 with Cryptosporidium spp. oocysts detected by modified Ziehl-Neelsen staining.

These Cryptosporidium spp. isolates had been previously genotyped by polymerase chain reaction-restriction fragment length polymorphism (PCR-RFLP) at the small subunit (SSU)-rRNA locus (Alves et al. 2001): 38 were identified as $C$. parvum and 23 as $C$. hominis. There were samples with low, moderate, and high parasite load. Samples positive for G. lamblia, C. parvum, and C. hominis were stored at $4{ }^{\circ} \mathrm{C}$ in an equal volume of $5 \%$ potassium dichromate solution for several years.

Eighteen water supply samples recovered from the environment by the United States Environmental Protection Agency (USEPA) Method 1623 (USEPA 1998), between
2003 and 2005 , and kept at $4^{\circ} \mathrm{C}$, were analyzed retrospectively: all samples were positive for Giardia spp. and Cryptosporidium spp. USEPA Method 1623 requires the concentration of water samples by filtration, immunomagnetic separation of the (oo)cysts from concentrated debris, detection of (oo)cysts by immunofluorescent assay, and viability determination by 4'-6-diamidino-2-phenylindole (DAPI).

Identification of Cryptosporidium spp. present in water supply Oocysts recovered from water samples after the immunomagnetic separation were submitted to DNA extraction by a MiniBeadBeaters/Silica method. Then, the species identification of these parasites was performed by PCR and by sequencing of the SSU-rRNA amplicon (Alves et al. 2001).

Oligonucleotide probes Three oligonucleotide probes, species-specific complementary to the $18 \mathrm{~S}$ rRNA region, were used for the FISH technique. Two probes specific for G. lamblia were used: Giar-4 (5'-CGGCGGGGGGCCAAC TAC-3') and Giar-6 (5'-CGGGGCTGCCGCGGCGCG-3'; Dorsch and Veal 2001). A C. parvum- and C. hominis-specific oligonucleotide probe, CRY-1 (5'-CGGTTATCCATGTAAG TAAAG-3'), was used (Vesey et al. 1998). The Giar-4, Giar-6, and CRY-1 oligonucleotide probes were synthesized by Thermo Electron $\mathrm{GmbH}$ in a $0.2-\mu \mathrm{M}$ scale, purified using reverse-phase high-performance liquid chromatography, and labeled with a single molecule of fluorochrome hexachlorinated 6-carboxyfluorescein (HEX) to the $5^{\prime}$ end.

MAbs An FITC-conjugated combination of MAbs specific to the cell wall antigens of Giardia spp. and Cryptosporidium spp. from the MerifluorTM Cryptosporidium/ Giardia test kit (Meridian Bioscience, Inc., 3471 River Hills, Cincinnati, OH, USA) was used.

FISH and FITC-conjugated MAbs in human fecal and water supply samples Fluorescent in situ hybridization and FITC-conjugated MAbs method was carried out as described previously (Graczyk et al. 2003). Hybridization protocols were optimized to get better results in fecal and water samples. Hence, the final concentration of each oligonucleotide probe used in human fecal and water supply samples was 2 and $10 \mathrm{pmol} / \mu \mathrm{l}$, respectively. Also, the final volume of fecal and water samples analyzed was 500 and $100 \mu l$, respectively.

Epifluorescence microscopy The wells were covered and examined with an Olympus epifluorescence microscope with $\times 40$ and $\times 100$ oil immersion objective lenses, and a BP-490 exciter filter was used.

Species confirmation of Giardia spp. parasites by molecular methods All FISH and FITC-conjugated MAbs fecal and water samples positive for Giardia spp. cysts were submitted to genomic DNA extraction. Cysts were concentrated from whole feces by a modified water-ether sedimentation method, and genomic DNA was isolated 
Table 1 Results of FISH and FITC-conjugated MAbs method on the identification and determination of the viability of (oo)cysts in the fecal and water supply samples parasitologically positive for Giardia spp. and Cryptosporidium spp. and confirmation of specificity of the probes used by molecular methods

\begin{tabular}{|c|c|c|c|c|c|}
\hline \multirow[t]{2}{*}{$\begin{array}{l}\text { Type of } \\
\text { samples } \\
\text { studied }\end{array}$} & \multicolumn{2}{|c|}{ Parasitological results } & \multicolumn{2}{|l|}{$\begin{array}{l}\text { Results of FISH } \\
\text { and FITC-conjugated } \\
\text { MAbs method }\end{array}$} & \multirow[t]{2}{*}{$\begin{array}{l}\text { Species identification } \\
\text { by molecular } \\
\text { methods }(n)\end{array}$} \\
\hline & $\begin{array}{l}\text { Protozoa } \\
\text { identified }\end{array}$ & $\begin{array}{l}\text { No. of } \\
\text { positive } \\
\text { samples }\end{array}$ & Positive samples (\%) & $\begin{array}{l}\text { Negative } \\
\text { samples (\%) }\end{array}$ & \\
\hline \multirow[t]{3}{*}{ Feces } & Giardia spp. & 50 & $9(18)$ & $41(82)$ & G. lamblia (3) \\
\hline & $\begin{array}{l}\text { Cryptosporidium } \\
\text { spp. }\end{array}$ & 61 & $3(5)$ & $58(95)$ & C. parvum (2) \\
\hline & & & & & C. hominis (1) \\
\hline \multirow[t]{3}{*}{ Water } & Giardia spp. & 18 & $6(33)$ & $12(67)$ & G.lamblia (1) \\
\hline & $\begin{array}{l}\text { Cryptosporidium } \\
\text { spp. }\end{array}$ & 18 & $2(11)$ & $16(89)$ & C. parvum (7)* \\
\hline & & & & & $\begin{array}{l}\text { C. parvum or } \\
\text { C. hominis }(2)^{*} \\
\text { C. muris }(1)^{*}\end{array}$ \\
\hline
\end{tabular}

*Samples found negative by FISH and FITC-conjugated MAbs method

samples positive parasitologically for G. lamblia, $9(18 \%)$ had viable cysts detected by FISH with MAb. Of the 61 fecal samples with C. parvum and C. hominis, $3(5 \%)$ had viable oocysts detected by FISH and FITC-conjugated MAb (Table 1). Eighteen water supply samples were analyzed by FISH with MAb, 2 weeks to 5 months after collection and processing according to USEPA Method 1623. Six $(33 \%)$ of the 18 samples studied presented viable and nonviable Giardia spp. cysts, and $2(11 \%)$ out of the 18 samples had viable and nonviable Cryptosporidium spp. oocysts detected (Table 1).

Potentially viable (oo)cysts were clearly differentiated from nonviable ones by color. Viable (oo)cysts were represented by intact green shells and were filled out completely with red cytoplasm, and the viable (oo)cysts were red inside as a result of FISH and had a green shell as a
Fig. 1 Distribution of the viable $G$. lamblia cysts in fecal samples between 2000 and 2004

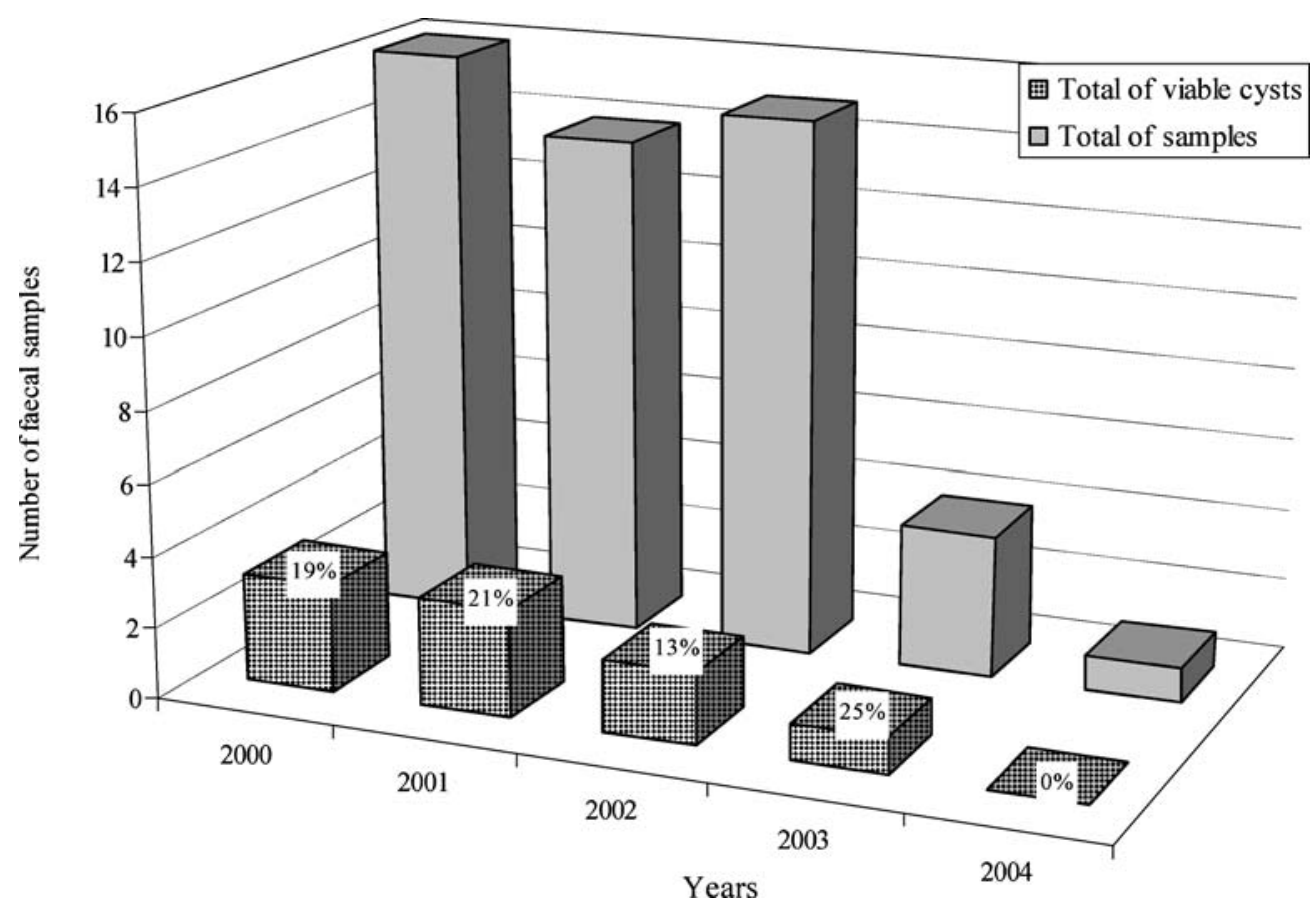


Fig. 2 Distribution of the viable Cryptosporidium spp. oocysts in fecal samples between 1997 and 2004

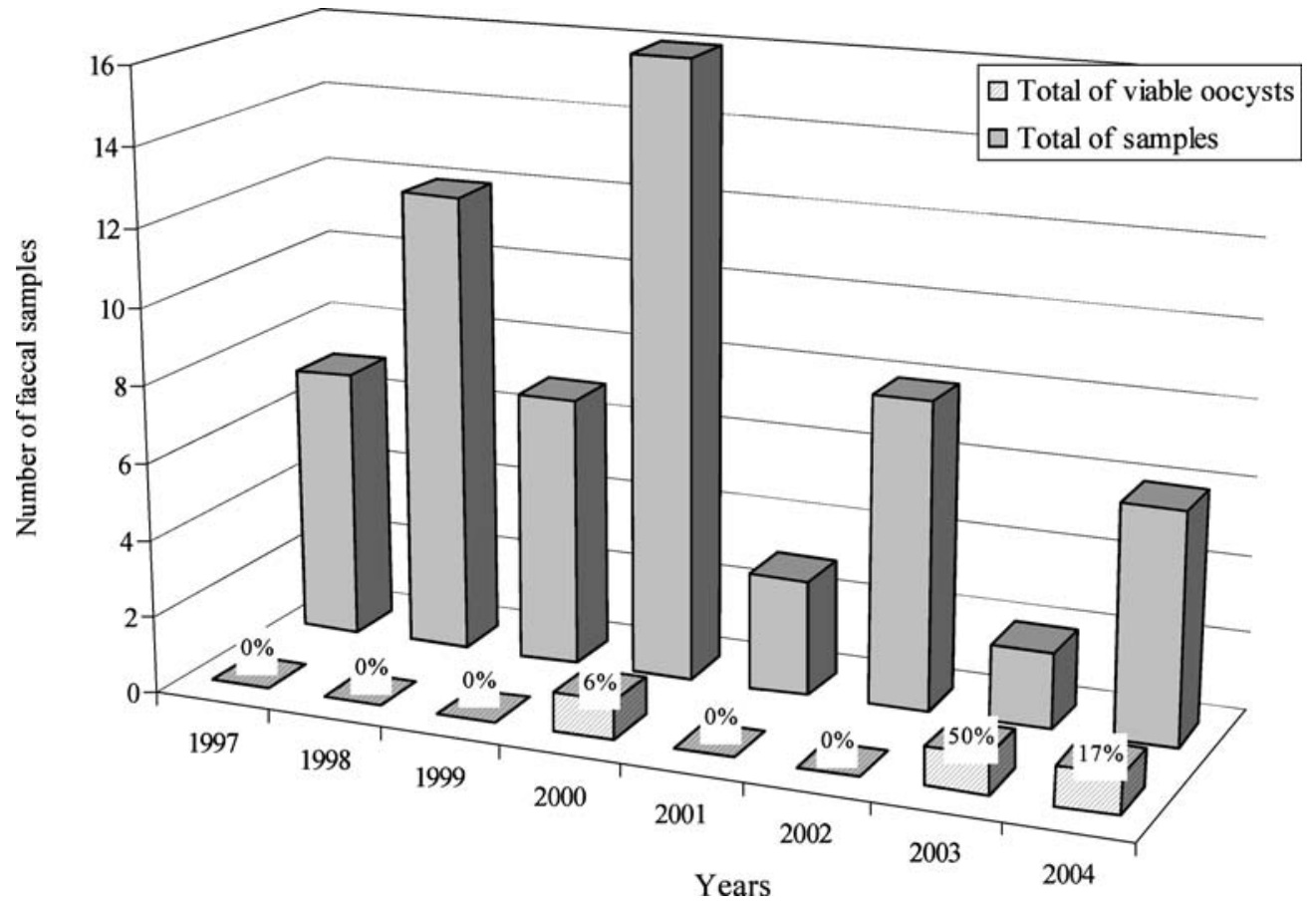

result of MAbs, as described previously (Graczyk et al. 2003).

With FISH and FITC-conjugated MAbs, viable (oo) cysts were detected in fecal samples with high, moderate, and low parasite loads. Viable Giardia spp. cysts were detected in fecal samples collected from 2000 until 2003 (Fig. 1), and viable Cryptosporidium spp. oocysts in fecal samples were recovered in 2000, 2003, and 2004 (Fig. 2). The highest percentage of viable (oo)cysts in feces was detected in 2003 (Figs. 1 and 2).
In the water supply samples studied, viable cysts (Fig. 3) were detected in 2003, 2004, and 2005, and viable oocysts were identified only in 2005 .

Identification of Cryptosporidium spp. present in water supply All 18 water samples analyzed by FISH and FITCconjugated MAbs, independent of being positive or negative, were submitted to PCR and sequencing at the SSU-rRNA locus. Isolates in 10 of the 18 water samples found positive by USEPA Method 1623 were successfully
Fig. 3 Distribution of the viable Giardia spp. cysts and Cryptosporidium spp. oocysts in water supply samples between 2003 and 2005

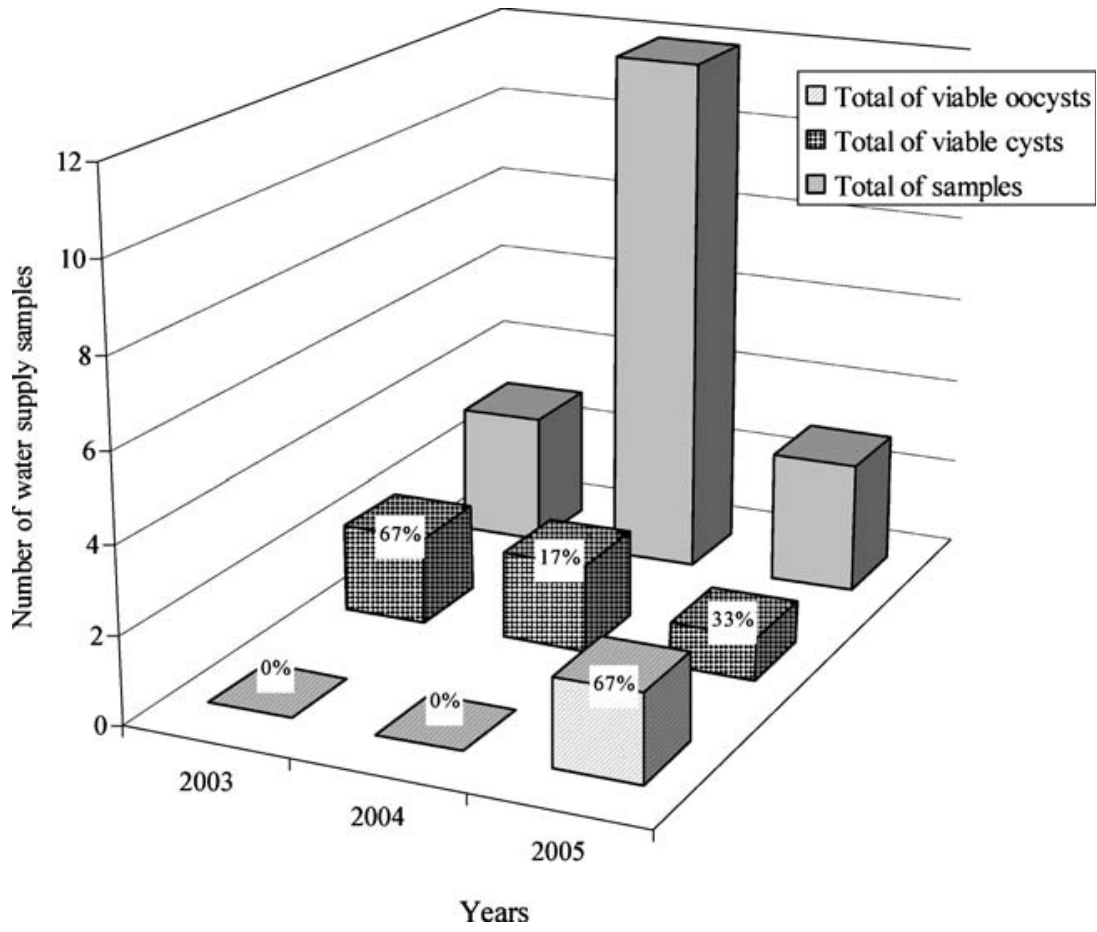


identified to the species level. C. parvum was implicated in the contamination of seven samples and Cryptosporidium muris in one. In two samples, the quality of the sequencing was poor and the sequence obtained could not differentiate between C. parvum and C. hominis. None of these samples was found positive by FISH and FITC-conjugated MAbs. The oligonucleotide probe used to detect $C$. parvum and $C$. hominis did not detect $C$. muris.

Species confirmation of Giardia spp. parasites by molecular methods Confirmation of Giardia spp. in fecal and water samples found positive by FISH and FITC-conjugated MAbs was achieved by PCR amplification of the $\beta$-giardin gene locus, followed by sequencing. This locus was successfully amplified in five fecal and four water samples. The sequence analysis was successfully obtained on the amplicons of the isolates of three fecal samples and of one water sample revealing $100 \%$ identity with $G$. lamblia Portland-1 (GenBank accession numbers: XO7919, X85958, $\mathrm{X} 14185$, and M36728).

\section{Discussion}

In the present study, the main objective was to determine if FISH and FITC-conjugated MAbs could have a role in the specific detection and viability determination of environmental parasites, voided in feces of animals or humans, more specifically G. lamblia, C. parvum, and C. hominis in water supply samples. This method was primarily applied to human fecal samples stored at $4^{\circ} \mathrm{C}$ for several years and lately applied to water supply samples stored for 2 weeks to 5 months. With FISH and FITC-conjugated MAbs, 9 $(18 \%)$ out of 50 human feces parasitologically positive for G. lamblia and $3(5 \%)$ out of 61 feces parasitologically positive for C. parvum and C. hominis presented viable and nonviable cysts and oocysts, respectively. A greater amount of nonviable (oo)cysts were detected in the oldest fecal samples, corroborating the results obtained in other studies (Jenkins et al. 2003; Surl et al. 2003). Viable Giardia spp. cysts and Cryptosporidium spp. oocysts were obtained since 2000 , corroborating also the idea that both protozoa can remain infectious over the years since they are very resistant in adverse environmental conditions.

After the successful application of this method in fecal samples, it was used to analyze 18 water supply samples positive for Giardia spp. and Cryptosporidium spp. by USEPA Method 1623. Viable and nonviable cysts of $G$. lamblia were identified in six (33\%) water samples, and oocysts of $C$. parvum and $C$. hominis were detected in two $(11 \%)$ water samples.

In general, more viable protozoa were found in feces than in water supply samples, which may be due to the higher parasite load, ease in detection, presence in feces than in water, and/or the small volume of water supply samples analyzed in this study. Also, G. lamblia cysts were detected in older samples (fecal and water samples), as against Cryptosporidium spp. oocysts. Viable Cryptospo- ridium spp. oocysts were detected only in the water samples analyzed by FISH and FITC-conjugated MAbs, the maximum of which was 2 weeks after recovery from the environment. Oocysts are probably more sensitive to manipulation by USEPA Method 1623 than cysts. Water samples must be submitted to FISH and FITC-conjugated MAbs for Giardia spp. and Cryptosporidium spp. immediately after recovery by the USEPA methods to overcome this problem, and a larger amount of water sample should be analyzed by this method.

In samples with Giardia spp., the molecular methods used to confirm the specificity of FISH and FITC-conjugated MAbs showed that the oligonucleotide probes used to detect G. lamblia are specific for this species. The oligonucleotide probe used to detect C. parvum and C. hominis also showed to be specific for these two species, as it did not detect $C$. muris in one water sample with this species. Nevertheless, it can also be due to loss of integrity of the oocysts since this was one of the samples studied by FISH and FITC-conjugated MAbs 1 month after its recovery from the environment and storage at $4^{\circ} \mathrm{C}$.

Contaminated water with G. lamblia, C. parvum, and $C$. hominis is an important source of human infection, either by direct consumption or by the use of contaminated water in food processing or preparation. Water transports transmissible stages into drinking water supplies, which, in turn, can contaminate the food supply through agricultural and food industry practices. In Portugal, although G. lamblia and Cryptosporidium spp. are recognized as common parasites in humans, little information on the prevalence and identity of these parasites' isolates from water is available.

With FISH and FITC-conjugated MAbs, the percentage of Cryptosporidium spp. oocysts found in water samples contaminated with oocysts of this parasite was low, suggesting that the practical application of FISH assay in water samples will require the development of methods capable of concentrating particulates from large volumes of water without affecting protozoa integrity.

FISH and FITC-conjugated MAbs provide information about the presence, the number, as well as the morphology and viability of the microorganism species involved in the contamination.

In conclusion, this study corroborates other opinions (Vesey et al. 1998; Graczyk et al. 2003; Smith et al. 2004) showing that FISH and FITC-conjugated MAbs is a highly specific and a sensitive assay for the detection of viable protozoa in fecal and environmental samples. Being a fast and cheap method, it combines the precision of molecular genetics with the visualization and identification of protozoa in comparison with parasitological methods, used for diagnosis and morphological studies, combined with PCRRFLP or DNA sequencing for species determination and with reverse transcriptase PCR for the evaluation of viability of microorganisms. Besides, this study sets the framework for further work on the presence of G. lamblia and Cryptosporidium spp. contamination in water and its importance in the field of public health in Portugal. 
Acknowledgments This work was supported by the Project POCTI/ESP/46369/2002 from Fundação para a Ciência e Tecnologia (FCT)/EU/FSE/FEDER and by a NATO Collaborative Linkage Grant, CLG 979765.

\section{References}

Alves M, Matos O, Antunes F (2001) Multilocus PCR-RFLP analysis of Cryptosporidium isolates from HIV-infected patients from Portugal. Ann Trop Med Parasitol 95:627-632

Amann RI, Ludwig W, Schleifer KH (1995) Phylogenetic identification and in situ detection of individual microbial cells without cultivation. Microbiol Rev 59:143-169

Caccio SM, De Giacomo M, Pozio E (2002) Sequence analysis of the beta-giardin gene and development of a polymerase chain reaction-restriction fragment length polymorphism assay to genotype Giardia duodenalis cysts from human faecal samples. Int J Parasitol 32:1023-1030

Dorsch MR, Veal DA (2001) Oligonucleotide probes for specific detection of Giardia lamblia cysts by fluorescent in situ hybridization. J Appl Microbiol 90:836-842

Graczyk TK, Grimes BH, Knight R, Da Silva AJ, Pieniazek NJ, Veal DA (2003) Detection of Cryptosporidium parvum and Giardia lamblia carried by synanthropic flies by combined fluorescent in situ hybridization and a monoclonal antibody. Am J Trop Med Hyg 68:228-232

Jenkins M, Trout JM, Higgins J, Dorsch M, Veal D, Fayer R (2003) Comparison of tests for viable and infectious Cryptosporidium parvum oocysts. Parasitol Res 89:1-5

Mac Kenzie WR, Hoxie NJ, Proctor ME, Gradus MS, Blair KA, Peterson DE, Kazmierczak JJ, Addiss DG, Fox KR, Rose JB, Davis JP (1994) A massive outbreak in Milwaukee of Cryptosporidium infection transmitted through the public water supply. N Engl J Med 331:161-167
Matos O, Tomás A, Aguiar P, Casemore D, Antunes F (1998) Prevalence of cryptosporidiosis in AIDS patients with diarrhoea in Santa Maria Hospital, Lisbon. Folia Parasitol 45:62-65

Moter A, Gobel UB (2000) Fluorescence in situ hybridization (FISH) for direct visualization of microorganisms. J Microbiol Methods 41:85-112

Ono K, Tsuji H, Rai SK, Yamamoto A, Masuda K, Endo T, Hotta H, Kawamura T, Uga S (2001) Contamination of river water by Cryptosporidium parvum oocysts in western Japan. Appl Environ Microbiol 67:3832-3836

Slifko TR, Smith HV, Rose JB (2000) Emerging parasite zoonoses associated with water and food. Int J Parasitol 30:1379-1393

Smith JJ, Gunasekera TS, Barardi CR, Veal D, Vesey G (2004) Determination of Cryptosporidium parvum oocyst viability by fluorescence in situ hybridization using a ribosomal RNAdirected probe. J Appl Microbiol 96:409-417

Solo-Gabriele HM, Leroy Ager A Jr, Fitzgerald LJ, Dubon JM, Neumeister SM, Baum MK, Palmer CJ (1998) Occurrence of Cryptosporidium oocysts and Giardia cysts in water supplies of San Pedro Sula, Honduras. Rev Panam Salud Publica 4:398400

Surl CG, Kim SM, Kim HC (2003) Viability of preserved Cryptosporidium baileyi oocysts. Korean J Parasitol 41:197-201

Tzipori S, Ward H (2002) Cryptosporidiosis: biology, pathogenesis and disease. Microbes Infect 4:1047-1058

United States Environmental Protection Agency (1998) Consumer confidence reports final rule. Fed Regist 63:160

Vesey G, Ashbolt N, Fricker EJ, Deere D, Williams KL, Veal DA, Dorsch M (1998) The use of a ribosomal RNA targeted oligonucleotide probe for fluorescent labelling of viable Cryptosporidium parvum oocysts. J Appl Microbiol 85:429-440 